\title{
GAIA Congenital Microcephaly Level of Diagnostic Certainty Terminology
}

National Cancer Institute

\section{Source}

National Cancer Institute. GAIA Congenital Microcephaly Level of Diagnostic Certainty

Terminology. NCI Thesaurus. Code C128700.

A subset of terminology related to congenital microcephaly, developed by the Global Alignment of Immunization safety Assessment in pregnancy consortium to aid in monitoring and improving fetal and maternal outcomes. 